\title{
Surface tension and energy conservation in a moving fluid
}

\author{
Bohr, Tomas; Scheichl, Bernhard
}

Published in:

Physical Review Fluids

Link to article, DOI:

10.1103/PhysRevFluids.6.L052001

Publication date:

2021

Document Version

Publisher's PDF, also known as Version of record

Link back to DTU Orbit

Citation (APA):

Bohr, T., \& Scheichl, B. (2021). Surface tension and energy conservation in a moving fluid. Physical Review Fluids, 6(5), [L052001 ]. https://doi.org/10.1103/PhysRevFluids.6.L052001

\section{General rights}

Copyright and moral rights for the publications made accessible in the public portal are retained by the authors and/or other copyright owners and it is a condition of accessing publications that users recognise and abide by the legal requirements associated with these rights.

- Users may download and print one copy of any publication from the public portal for the purpose of private study or research.

- You may not further distribute the material or use it for any profit-making activity or commercial gain

- You may freely distribute the URL identifying the publication in the public portal

If you believe that this document breaches copyright please contact us providing details, and we will remove access to the work immediately and investigate your claim 


\title{
Surface tension and energy conservation in a moving fluid
}

\author{
Tomas Bohr ${ }^{1, *}$ and Bernhard Scheichl $\odot^{2,3, \dagger}$ \\ ${ }^{1}$ Department of Physics, Technical University of Denmark, DK-2800 Kgs. Lyngby, Denmark \\ ${ }_{2}^{2}$ Institute of Fluid Mechanics and Heat Transfer, Technische Universität Wien, \\ Getreidemarkt 9, 1060 Vienna, Austria \\ ${ }^{3} A C 2 T$ research GmbH, Viktor-Kaplan-Straße 2/C,2700 Wiener Neustadt, Austria
}

(Received 8 December 2020; accepted 27 April 2021; published 28 May 2021)

\begin{abstract}
The transport of energy in a moving fluid with a simply connected free surface is analyzed, taking into account the contribution of surface tension. This is done by following a "control volume" with arbitrary, specified velocity, independent of the flow velocity, and determining the rates of energy passing through the boundaries, as well as the energy dissipation in the bulk. In particular, a simple conservation equation for the surface area is given, which clearly shows the contribution of the Laplace pressure at the free surface and the tangential surface tension forces at its boundary. It emerges as the mechanical conservation law for the surface energy in its general form. For a static control volume, all contributions from surface tension disappear, except that the pressure has to be modified by the Laplace contribution.
\end{abstract}

DOI: 10.1103/PhysRevFluids.6.L052001

Capillary effects have been included in flows with free surfaces or interfaces for around 150 years. Some famous pioneers are Rayleigh [1,2], Landau and Levich [3], Taylor [4], Culick [5], and Bretherton [6], and it is still an active field of research with outstanding survey papers [7,8] and the textbook [9]. In contrast to flows having free surfaces with objects protruding, where surface tension gives rise to tangential forces at the boundaries, or Marangoni flows, where gradients of the surface tension contribute to the surface-stress balance, free-surface flows with uniform surface tension experience only a discontinuity of the normal component of the fluid stresses on the interface, commonly referred to as Laplace pressure and described, e.g., carefully by Batchelor [10] (Sec. 1.9).

Although this is merely a consequence of the governing equations of motion and conventional kinematic and dynamic boundary conditions, this procedure has been challenged recently in Journal of Fluid Mechanics by Bhagat et al. [11] and Bhagat and Linden [12]. These authors claim that the tangential surface tension contributes to a power term in the integral energy budget and should, therefore, be taken into account explicitly. For a static control volume in a steady (stationary) flow, this power is claimed to be

$$
\oint_{\mathcal{C}_{s}} \mathbf{f}_{\gamma} \cdot \mathbf{u} d \mathcal{C}_{s}
$$

Here the closed curve $\mathcal{C}_{s}$ has the infinitesimal arc length $d \mathcal{C}_{s}$ and encloses a portion of the free surface that forms the control area and confines the control volume, $\mathbf{u}$ is the flow velocity on $\mathcal{C}_{s}$, and $\mathbf{f}_{\gamma}$ is the external surface tension force (per length). Indeed, inclusion of such a power term,

\footnotetext{
*tomas.bohr@fysik.dtu.dk

†bernhard.scheichl@tuwien.ac.at
} 


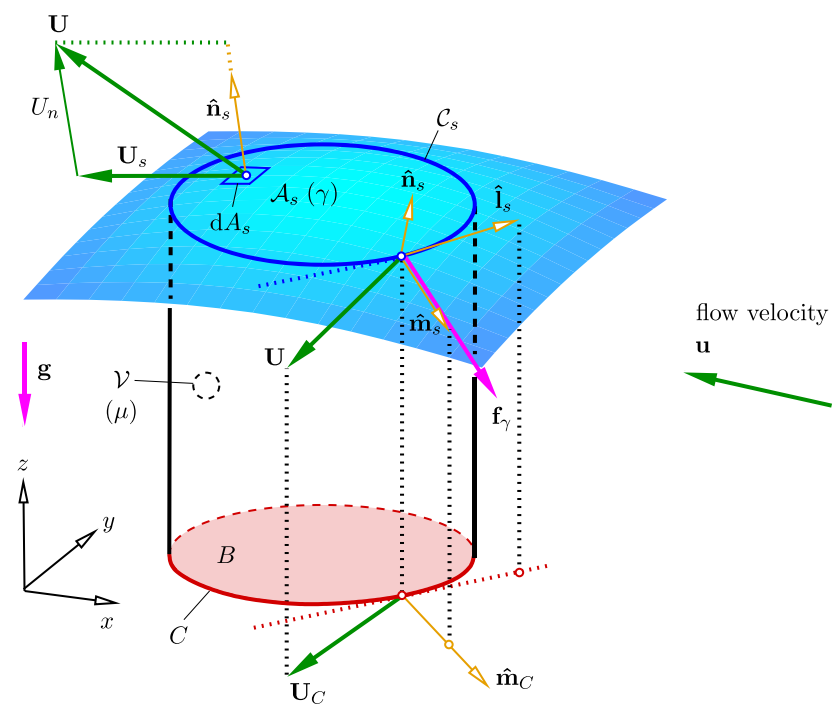

FIG. 1. Control volume $\mathcal{V}$ of bulk flow, moving with speed $\mathbf{U}$ in a flow with velocity $\mathbf{u}$, bounded by control area $\mathcal{A}_{s}$ of free surface (top face, blue) enclosed by $\mathcal{C}_{s}$, projections $B, C$ of $\mathcal{A}_{s}, \mathcal{C}_{s}$ onto $(x, y)$ plane (bottom, red), and projection lines (bold black); essential bulk and surface properties $(\mu, \gamma)$, body and surface force densities (magenta), velocities (green), unit vectors (empty arrows, orange); dotted: tangents and projection lines of vectors.

referring to the external work of surface tension, seems intuitively correct and its omission therefore a serious error in all the preceding works. However, in this paper we demonstrate that within the continuum hypothesis, where the interface is taken as infinitely thin and mass- and inertialess, this is not the case. Such a power contribution is present only when the control surface is moving, and then the velocity $\mathbf{u}$ in (1) should be replaced by the velocity of the control surface, $\mathbf{U}$, transporting $\mathcal{C}_{s}$. Even in the general situation of an unsteady flow, the appearance of this term is inconsistent with the advection of surface energy combined with the standard work-energy balance for the bulk flow, derived from the momentum (Navier-Stokes) equations. This was already noticed by Duchesne et al. [13] and Scheichl [14], but in the present paper we show how this can be seen directly from the conservation law for a moving surface area that we derive below.

We consider a free-surface flow of an incompressible fluid (liquid) of uniform density $\rho$, as sketched in Fig. 1. The free surface forming the interface with the (gaseous) environment is characterized by a uniform surface tension $\gamma$. The environment is typically taken as nominally at rest and under a constant ambient pressure $p_{0}$. We assume that the dynamic viscosity $\mu$ for the gas is much smaller than that of the liquid, so the deviatoric stresses from the external gas flow can be neglected (for simplicity). Let $t$ denote the time and $\mathbf{x}=x \hat{\mathbf{x}}+y \hat{\mathbf{y}}+z \hat{\mathbf{z}}=(x, y, z)$ the space vector expressed in static Cartesian laboratory coordinates $x, y, z$ and the associated unit vectors (indicated with hats). For partial derivatives we use $\nabla=\left(\partial_{x}, \partial_{y}, \partial_{z}\right)$ and $\partial_{t}$ as well as subscripts $t, x, y, z$ of scalars that cannot be confused with indices. We assume gravity to be the only body force at play, with the constant gravitational acceleration $\mathbf{g}=(0,0,-g)$. In the bulk of the flow, the Eularian "material" velocity field $\mathbf{u}(\mathbf{x}, t)=(u, v, w)$, the pressure $p(\mathbf{x}, t)$, and the (symmetric) tensor $\mathbf{T}$ of the deviatoric (viscous) Cauchy stresses, subject to a constitutive relationship, then satisfy the continuity and momentum equations

$$
\begin{gathered}
\nabla \cdot \mathbf{u}=0 \\
\rho\left(\partial_{t} \mathbf{u}+\mathbf{u} \cdot \nabla \mathbf{u}\right)=-\nabla p-\rho g \hat{\mathbf{z}}+\nabla \cdot \mathbf{T} .
\end{gathered}
$$


For a Newtonian liquid with the dynamic viscosity $\mu, \mathbf{T}=\mu \mathbf{S}$ where $\mathbf{S}=\left[\nabla \mathbf{u}+(\nabla \mathbf{u})^{T}\right]$ is twice the strain-rate tensor.

We look at the simply connected control area $\mathcal{A}_{s}$ of the moving fluid surface with the boundary $\mathcal{C}_{s}=\partial \mathcal{A}_{s}$ and consider also their projections $B$ and $C=\partial B$ on to the $(x, y)$ plane (the "bottom"). The free surface is described simplistically as a function $z=h(x, y, t)$. The value of $\mathbf{u}$ on the surface can be decomposed into a component in the surface (i.e., in the tangent plane of the instantaneous surface) $\mathbf{u}_{s}(x, y, t)$ and a normal component $u_{n}(x, y, t)$ along the outward unit normal vector

$$
\hat{\mathbf{n}}_{s}(x, y, t)=\frac{\left(-h_{x},-h_{y}, 1\right)}{\sqrt{1+|\nabla h|^{2}}}=\frac{\hat{\mathbf{z}}-\nabla h}{\sqrt{1+|\nabla h|^{2}}} .
$$

We furthermore introduce the vectors

$$
\hat{\mathbf{l}}_{s}=d \mathbf{x} / d \mathcal{C}_{s}, \quad \hat{\mathbf{m}}_{s}=\hat{\mathbf{l}}_{s} \times \hat{\mathbf{n}}_{s}, \quad \mathbf{f}_{\gamma}=\gamma \hat{\mathbf{m}}_{s}
$$

where $d \mathcal{C}_{s}$ is the infinitesimal arc length on $\mathcal{C}_{s}, \hat{\mathbf{l}}_{s}$ a unit tangent vector on $\mathcal{C}_{s}, \hat{\mathbf{m}}_{s}$ is a unit vector perpendicular to $\mathcal{C}_{s}$, and $\mathbf{f}_{\gamma}$ is the tangential capillary force per length from the surface region outside $\mathcal{C}_{s}$. Similarly, in the $(x, y)$ plane, with infinitesimal arc length $d C$ of $C$, its outer normal $\hat{\mathbf{m}}_{C}$, and the projection of $\hat{\mathbf{n}}_{s}$ are

$$
\hat{\mathbf{m}}_{C}=\frac{(d y,-d x, 0)}{d C}, \quad \mathbf{n}_{b}=\frac{-\nabla h}{\sqrt{1+|\nabla h|^{2}}} .
$$

Further $\mathbf{u}$ satisfies the kinematic free-surface boundary condition

$$
h_{t}=w-u h_{x}-v h_{y}=u_{n} \sqrt{1+|\nabla h|^{2}}
$$

on $\mathcal{A}_{s}$, so $u_{n}$ is only nonzero when $h$ is explicitly time-dependent. The dynamic boundary condition expresses continuity of the total stress across $\mathcal{A}_{s}$ in the form (see, e.g., [15], § 61)

$$
\left(p-p_{0}\right) \hat{\mathbf{n}}_{s}-\hat{\mathbf{n}}_{s} \cdot \mathbf{T}-\Delta p_{L} \hat{\mathbf{n}}_{s},
$$

where $\Delta p_{L}$ is the Laplace pressure involving (twice) the local mean curvature $\kappa$ of $\mathcal{A}_{s}$ as

$$
\Delta p_{L}=\gamma \kappa=\gamma \nabla \cdot \hat{\mathbf{n}}_{s}=\gamma \nabla \cdot \mathbf{n}_{b}
$$

(where the sign of $\kappa$ is chosen such that $\kappa>0$ and $\nabla^{2} h<0$ for a convex, droplet-shaped surface). The equivalence of the local stress equilibrium (8) on $\mathcal{A}_{s}$ with its global counterpart

$$
\int_{\mathcal{A}_{s}}\left[\left(p-p_{0}\right) \hat{\mathbf{n}}_{s}-\hat{\mathbf{n}}_{s} \cdot \mathbf{T}\right] d A_{s}=-\oint_{\mathcal{C}_{s}} \mathbf{f}_{\gamma} d \mathcal{C}_{s}
$$

is obtained using Stokes' theorem in the variant

$$
\oint_{\mathcal{C}_{s}} \hat{\mathbf{m}}_{s} d \mathcal{C}_{s}=\oint_{\mathcal{C}_{s}} \hat{\mathbf{l}}_{s} \times \hat{\mathbf{n}}_{s} d \mathcal{C}_{s}=-\int_{\mathcal{A}_{s}}\left(\nabla \cdot \hat{\mathbf{n}}_{s}\right) \hat{\mathbf{n}}_{s} d A_{s}
$$

as shown, e.g., in [16] (p. 235), with the counterintuitive result that an integral over tangential vectors points in the direction of the normals.

It is our goal to combine the (hitherto unappreciated) conservation law for the surface energy contained in $\mathcal{A}_{s}$ with that for the mechanical energy of the bulk flow in an arbitrarily moved, simply connected control volume $\mathcal{V}$ bounded by $\mathcal{A}_{s}$ at the free surface. We denote the velocity field moving the closed boundary $\partial \mathcal{V}$ as $\mathbf{U}(\mathbf{x}, t)=(U, V, W)$ and denote its component normal to $\mathcal{A}_{s}$ as $U_{n}$. Analogously, $C$ moves with $\mathbf{U}_{C}$, the projection of $\mathbf{U}$ evaluated on $\mathcal{C}_{s}$ on the $(x, y)$ plane. Most importantly, $\mathbf{U}$ must be kinematically compatible to the material flow field $\mathbf{u}$ in the sense that any point on the free surface considered to move with $\mathbf{U}$ remains in the surface for all times. That is, $\mathbf{U}$ satisfies

$$
h_{t}=W-U h_{x}-V h_{y}=U_{n} \sqrt{1+|\nabla h|^{2}}
$$


on $\mathcal{A}_{s}$, in analogy to (7). Thus a completely static control volume $\mathcal{V}$ is of course possible only for a steady flow.

The surface energy stored in $\mathcal{A}_{s}$ is simply $E_{s}=\gamma A_{s}$ where

$$
A_{s}=\int_{\mathcal{A}_{s}} d A_{s}=\int_{B} \sqrt{1+|\nabla h|^{2}} d x d y
$$

is the time-dependent area of $\mathcal{A}_{s}$.

The integral conservation law for the surface energy is found by analyzing $\gamma d A_{s} / d t$ in terms of $\mathbf{U}$, which transports $\mathcal{V}$ and thus $\mathcal{A}_{s}$. Using Leibnitz's integral rule, the time derivative of (13) is

$$
\frac{d A_{s}}{d t}=\int_{B} \partial_{t} \sqrt{1+|\nabla h|^{2}} d x d y+\oint_{C} \sqrt{1+|\nabla h|^{2}} \mathbf{U}_{C} \cdot \hat{\mathbf{m}}_{C} d C
$$

The first term to the right can be rewritten as

$$
\begin{aligned}
\int_{B} \frac{\nabla h_{t} \cdot \nabla h}{\sqrt{1+|\nabla h|^{2}}} d x d y & =\int_{B}\left[-h_{t} \nabla \cdot\left(\frac{\nabla h}{\sqrt{1+|\nabla h|^{2}}}\right)+\nabla \cdot\left(\frac{h_{t} \nabla h}{\sqrt{1+|\nabla h| 2}}\right)\right] d x d y \\
& =\int_{B} h_{t} \kappa d x d y+\oint_{C} \frac{h_{t}}{\sqrt{1+|\nabla h|^{2}}} \nabla h \cdot \hat{\mathbf{m}}_{C} d C .
\end{aligned}
$$

The last equality follows from (9) and the divergence theorem (or, more precisely, Green's theorem, since the integral of the divergence is over a surface). All in all, we have

$$
\frac{d A_{s}}{d t}=\int_{B} h_{t} \kappa d x d y+\oint_{C}\left(\sqrt{1+|\nabla h|^{2}} \mathbf{U}_{C}+\frac{h_{t}}{\sqrt{1+|\nabla h|^{2}}} \nabla h\right) \cdot \hat{\mathbf{m}}_{C} d C .
$$

The expressions (5) combined with (4) yield the explicit representation

$$
\hat{\mathbf{m}}_{s}=\frac{\left.\left[\left(1+h_{y}^{2}\right) d y,-\left(1+h_{x}^{2}\right) d x, h_{x} d y-h_{y} d x\right)\right]}{\sqrt{1+|\nabla h|^{2}} d \mathcal{C}_{s}},
$$

and together with the kinematic boundary condition (12) this leads to the simple result

$$
\frac{d A_{s}}{d t}=\int_{\mathcal{A}_{s}} U_{n} \kappa d A_{s}+\oint_{\mathcal{C}_{s}} \mathbf{U} \cdot \hat{\mathbf{m}}_{s} d \mathcal{C}_{s} .
$$

Actually, this result could have been obtained more directly and intuitively by considering independently the three infinitesimally small temporal (linear) variations of the shape of the material surface element $d A_{s}$ : (1) its dilatation normal to $\mathcal{A}_{s}$ (inflation), (2) its distortion in its tangential directions (stretching and shearing), and (3) its tangential rigid-body movement. Since the last has no effect, this leaves us with the two contributions on the right side of (18), where the first term originates in the relative differential variation of $d A_{s}$ in the normal direction, $\left(\delta_{n} d A_{s}\right) / d A_{s}$ (see, e.g., [17] and [15], § 61), and the last term describes the tangential net change of $A_{s}$ as the annihilation/generation of area adjacent to $\mathcal{C}_{s}$. In fact, these considerations immediately confirm the extension of the well-known Leibnitz-Reynolds transport theorem to the general transport of some scalar $f(\mathbf{x}, t)$ on a moving surface $\mathcal{A}_{s}$. Following, e.g., [18] [Eq. (3.14)] or [19] (Theorem 4.2), this can be written in the form

$$
\frac{d}{d t} \int_{\mathcal{A}_{s}} f(\mathbf{x}, t) d A_{s}=\int_{\mathcal{A}_{s}}\left(\partial_{t}+U_{n} \partial_{n}+U_{n} \kappa\right) f d A_{s}+\oint_{\mathcal{C}_{s}} f \mathbf{U} \cdot \hat{\mathbf{m}}_{s} d \mathcal{C}_{s},
$$

where $\partial_{n}=\hat{\mathbf{n}}_{s} \cdot \nabla$ is the normal component of $\nabla$ on $\mathcal{A}_{s}$. The first and the second integral on the right side of (19) account for, respectively, the surface-normal and tangential variations of both $A_{s}$ and $f$. Therefore, the first includes the area-fixed time derivative normal to the surface $\left(\partial_{t}+U_{n} \partial_{n}\right) f$. The succinct derivation of (18) follows from taking $f=$ const. 


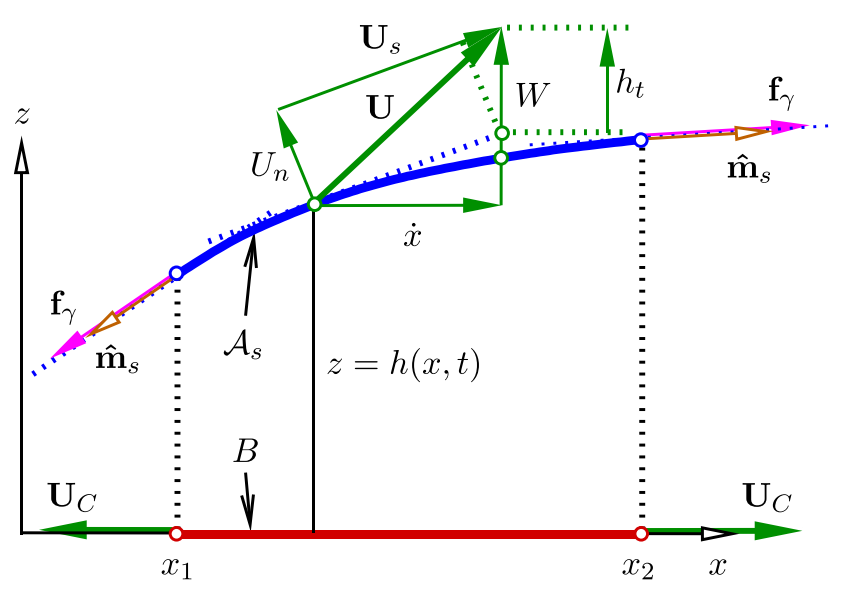

FIG. 2. Planar flow: projection onto $(x, z)$ plane; for legend see Fig. 1.

It is useful to also consider an alternative form containing only surface integrals. To this end, we introduce the tangential part $\nabla_{s}=\nabla-\hat{\mathbf{n}}_{s} \partial_{n}$ of $\nabla$, acting on the tangential flow field $\mathbf{U}_{s}=$ $\mathbf{U}-U_{n} \hat{\mathbf{n}}_{s}$. Using this notation, the divergence theorem for a surface [see Eq. (A.7.6) in [16], in the presence of a scalar field] can be written as

$$
\oint_{\mathcal{C}_{s}} f \mathbf{U} \cdot \hat{\mathbf{m}}_{s} d \mathcal{C}_{s}=\int_{\mathcal{A}_{s}} \nabla_{s} \cdot\left(f \mathbf{U}_{s}\right) d A_{s},
$$

where $\nabla_{s} \cdot \hat{\mathbf{n}}_{s}=\nabla \cdot \hat{\mathbf{n}}_{s}=\kappa[$ [Eq. (9)]. Then we can rewrite (19) first as

$$
\frac{d}{d t} \int_{\mathcal{A}_{s}} f(\mathbf{x}, t) d A_{s}=\int_{\mathcal{A}_{s}}\left[\partial_{t} f+U_{n} \partial_{n} f+U_{n} \kappa f+\nabla_{s} \cdot\left(f \mathbf{U}_{s}\right)\right] d A_{s}
$$

and, noticing that $\nabla_{s} U_{n}$ is tangential to the surface, in the form

$$
\frac{d}{d t} \int_{\mathcal{A}_{s}} f(\mathbf{x}, t) d A_{s}=\int_{\mathcal{A}_{s}}\left(\partial_{t} f+\mathbf{U} \cdot \nabla f+f \nabla_{s} \cdot \mathbf{U}\right) d A_{s} .
$$

Here the full substantial derivative $\partial_{t} f+\mathbf{U} \cdot \nabla f$ appears. This form, as well as (20), was given in [20] [Eqs. (A2) and (A4)].

It is instructive to write the analog of (18) for a planar flow where the free surface is a line $z=h(x, t)$ as shown Fig. 2. The control volume (area) has endpoints $x=x_{1}(t)$ and $x=x_{2}(t)>x_{1}$ on the free surface that move with the imposed velocity $U(x, t)$. The analog of the surface area, i.e., the length of the free surface segment, is

$$
L_{s}=\int_{\mathcal{L}_{s}} d L_{s}=\int_{x_{1}}^{x_{2}} \sqrt{1+h_{x}^{2}} d x
$$

and the curvature $\kappa_{1}$ is

$$
\kappa_{1}=-\frac{\partial}{\partial x}\left(\frac{h_{x}}{\sqrt{1+h_{x}^{2}}}\right)=-\frac{h_{x x}}{\left(1+h_{x}^{2}\right)^{3 / 2}} .
$$

If $d$ denotes its depth in the $y$ direction, $E_{s}=\gamma L_{s} d$ is the corresponding surface energy. In order to consider the advection of a point on this segment by a velocity field $\mathbf{U}(x, y, t)=(U, 0, W)$, we take its $x$ coordinate as time-dependent, thus its $z$ coordinate as $h(x(t), t)$. On $\mathcal{C}_{s}, U=\dot{x}$ and $W=h_{t}+\dot{x} h_{x}$ by (12). It is evident that $\mathbf{U} \cdot \hat{\mathbf{m}}_{s}$ vanishes on the points of $\mathcal{C}_{s}$ with $x$ coordinates located between $x_{1}$ and $x_{2}$. Here $\hat{\mathbf{m}}_{s}=\left(\hat{\mathbf{x}}+h_{x} \hat{\mathbf{z}}\right) / \sqrt{1+h_{x}^{2}}$ and $\mathbf{U}$ are collinear if $h_{t}$ vanishes. These 
results help us to rewrite (18) in this case as

$$
\begin{aligned}
\frac{d L_{s}}{d t} & =\int_{x_{1}}^{x_{2}} h_{t} \kappa_{1} d x+\left[\sqrt{1+h_{x}^{2}} \dot{x}+\frac{h_{t} h_{x}}{\sqrt{1+h_{x}^{2}}}\right]_{x=x_{1}}^{x=x_{2}} \\
& =\int_{\mathcal{L}_{s}} U_{n} \kappa_{1} d L_{s}+\left[\mathbf{U} \cdot \hat{\mathbf{m}}_{s}\right]_{x=x_{1}}^{x=x_{2}}
\end{aligned}
$$

in direct analogy with (16) by noticing the projection $\mathbf{U}_{C}=\dot{x}_{1,2} \hat{\mathbf{x}}$ of $\mathbf{U}$ onto the endpoints. Alternatively, one obtains (25) from (18) in straightforward manner under the assumptions of a planar flow and $\mathcal{C}$ chosen as a rectangle with a side of length $x_{2}-x_{1}$ parallel to the $x$ axis.

We next remark some important consequences of (18). First, we derive the differential version, using (22) for $f$ as a constant, and expanding $\nabla_{s} \cdot \mathbf{U}=\nabla_{s} \cdot \mathbf{U}_{s}+U_{n} \nabla_{s} \cdot \hat{\mathbf{n}}_{s}=\boldsymbol{\nabla} \cdot \mathbf{U}-\hat{\mathbf{n}}_{s} \cdot\left(\hat{\mathbf{n}}_{s} \cdot\right.$ $\nabla \mathbf{U})$. Then (18) takes on the form

$$
\frac{d A_{s}}{d t}=\int_{\mathcal{A}_{s}}\left[U_{n} \boldsymbol{\nabla} \cdot \hat{\mathbf{n}}_{s}+\nabla_{s} \cdot \mathbf{U}_{s}\right] d A_{s}=\int_{\mathcal{A}_{s}}\left[\nabla \cdot \mathbf{U}-\hat{\mathbf{n}}_{s} \cdot(\nabla \mathbf{U}) \cdot \hat{\mathbf{n}}_{s}\right] d A_{s} .
$$

As $\mathcal{A}_{s}$ is chosen arbitrarily, the relative temporal rate of change of an infinitesimal area element must be

$$
\left(\delta d A_{s} / \delta t\right) / d A_{s}=U_{n} \nabla \cdot \hat{\mathbf{n}}_{s}+\nabla_{s} \cdot \mathbf{U}_{s}=\nabla \cdot \mathbf{U}-\hat{\mathbf{n}}_{s} \cdot(\nabla \mathbf{U}) \cdot \hat{\mathbf{n}}_{s} .
$$

For $\mathbf{U}$ identical to the material flow field $\mathbf{u}$, this local form of (26) was already derived by Batchelor [10], Eqs. (3.1.5)-(3.1.8), without taking notice of its integral formulation. For an incompressible material flow field, it takes the form $\left(\delta d A_{s} / \delta t\right) / d A_{s}=-\hat{\mathbf{n}}_{s} \cdot(\nabla \mathbf{u}) \cdot \hat{\mathbf{n}}_{s}$ and these local forms makes explicit the required invariance under the Galilean boost, i.e., for (27): $\mathbf{U} \mapsto \mathbf{U}+\mathbf{C}, \mathbf{x} \mapsto \mathbf{x}+\mathbf{C} t$, which is evident neither from (18) nor from (16).

Finally, for a stationary control volume velocity $\mathbf{U}$, the curvature term in (18) is zero. But interestingly and counterintuitively, $d A_{s} / d t$ does not necessarily vanish for a control volume following the flow (i.e., $\mathbf{U}=\mathbf{u}$ ) even for a stationary incompressible flow. Incompressibility suppresses the dilatation of the fluid particles but not their stretching and shearing in the surface governed by the surface divergence in (20). Therefore, the streamlines lying in the surface can diverge/converge, which explains the in general nonvanishing $d A_{s} / d t$.

In the remainder of this letter, we discuss the dynamic implications of the kinematic relationship (18). Multiplying (18) with $\gamma$ and using the expressions for the Laplace pressure in (8) and the vectorial surface tension, $\mathbf{f}_{\gamma}=\gamma \hat{\mathbf{m}}_{C}$, results in the integral transport equation for the surface energy convected by $\mathbf{U}$ :

$$
\frac{d E_{s}}{d t}=\int_{\mathcal{A}_{s}} \Delta p_{L} U_{n} d A_{s}+\oint_{\mathcal{C}_{s}} \mathbf{f}_{\gamma} \cdot \mathbf{U} d \mathcal{C}_{s} .
$$

This has an appealing and convincing physical interpretation as a law of mechanical energy conversion for the piece $\mathcal{A}_{s}$ of the inertialess free fluid surface: the work done by the Laplace pressure on $\mathcal{A}_{s}$ and by the tangential surface tension forces at its boundary $\mathcal{C}_{s}$, on the surface moving with the imposed velocity field $\mathbf{U}$, changes the potential internal energy, i.e., the surface energy $E_{S}$, contained in $\mathcal{A}_{s}$. Therefore, (28) represents the precise justification for the Laplace pressure and the existence of the tangential surface tension forces. Note that the inclusion of the Marangoni effect (inhomogeneous $\gamma$ ) would lead to an extension of (28) incorporating the transport of $\gamma$ as a nonuniform passive scalar: see (19), (21), and (22) with $f=\gamma(\mathbf{x}, t)$. Differential versions are given in Eqs. (5) and (6) of [21] describing the transport of a surfactant on a deforming fluid surface: a nonconstant surface tension then is a field quantity defined only on the free surface, so $\partial_{n} \gamma$ must be taken as zero.

The conservation law (28) is the basis for the subsequent careful re-examination of the workenergy balance for the entire volume $\mathcal{V}$ including its top face $\mathcal{A}_{s}$ once $\mathbf{U}$ is specified appropriately. To do this, we first briefly recall the derivation of the classical work-energy theorem as stated, e.g., 
by Eq. (16.2) in [15]. More precisely, it denotes the transport of the overall mechanical energy of the bulk flow,

$$
E_{m}=\int_{\mathcal{V}} e_{m} d V, \quad e_{m}=\frac{\rho|\mathbf{u}|^{2}}{2}+\rho g z
$$

where the energy density $e_{m}$ consists of the kinetic and the potential energy densities. Taking the inner product of $\mathbf{u}$ and the momentum equation (3) together with the incompressibility condition (2) and the identity $w=\nabla \cdot(z \mathbf{u})$ leads to the differential form of the energy equation:

$$
\partial_{t} e_{m}=\nabla \cdot\left[\mathbf{T} \cdot \mathbf{u}-\left(p+e_{m}\right) \mathbf{u}\right]-\mathbf{T}: \nabla \mathbf{u} .
$$

Integration of (30) over $\mathcal{V}$ and applying the divergence theorem gives

$$
\int_{\mathcal{V}} \partial_{t} e_{m} d V=\oint_{\partial \mathcal{V}}\left[\mathbf{T} \cdot \mathbf{u}-\left(p+e_{m}\right) \mathbf{u}\right] \cdot \hat{\mathbf{n}} d A-\int_{\mathcal{V}} \mathbf{T}: \nabla \mathbf{u} d V
$$

where the first term represents the advected energy, and the last term represents the (non-negative) integral dissipation per time unit due to the deviatoric stresses, commonly denoted with $\dot{\Phi}$. We have also introduced the infinitesimal area element $d A$ of $\partial \mathcal{V}$ and its outward unit normal $\hat{\mathbf{n}}$. We note that, according to the derivation of (31), the stresses acting on $\partial \mathcal{V}$ are taken as their limiting values approached from inside $\mathcal{V}$. For the bulk flow this makes no difference due to continuity, but when we include the free surface it becomes important. The transport equation then follows from the classical Leibnitz-Reynolds transport theorem [cf. [22] and Eqs. (3.1.3)-(3.1.5) in [10]]:

$$
\begin{aligned}
\frac{d E_{m}}{d t} & =\int_{\mathcal{V}} \partial_{t} e_{m} d V+\oint_{\partial \mathcal{V}} e_{m} \mathbf{U} \cdot \hat{\mathbf{n}} d A \\
& =\oint_{\partial \mathcal{V}}\left[e_{m}(\mathbf{U}-\mathbf{u})+\mathbf{T} \cdot \mathbf{u}-p \mathbf{u}\right] \cdot \hat{\mathbf{n}} d A-\dot{\Phi}
\end{aligned}
$$

This work-energy principle states that the temporal change of $E_{m}$ is compensated by the flux of mechanical energy into $\mathcal{V}$ and the external work done by the total stresses acting on $\partial \mathcal{V}$, due to the material velocity $\mathbf{u}$, and the associated internal work of the deviatoric (viscous) stresses, equal to the negative mechanical dissipation. The left side of (32) equals zero for a stationary flow.

For many applications, especially for stationary flows, it is valuable to consider a cylindrical control volume $\mathcal{V}$ aligned with $z$ direction and having the patch $\mathcal{A}_{s}$ of the free surface as its top boundary, as indicated in Fig. 1. In the general, nonstationary flow situation its lateral sides facing the bulk of the flow and $\mathcal{A}_{s}$ are both moving. In the important case of a static control volume, however, its projection $B$ and thus $C$ stay stationary, while its top face $\mathcal{A}_{s}$ is moving with the normal component $u_{n}$ of the material flow (when the flow is not stationary), following from comparison of (12) with (7). Thus, $\mathbf{U}=\mathbf{0}$ on the lateral sides of $\mathcal{V}, \mathbf{U}_{C}=(U, V, 0) \equiv \mathbf{0}$, but $\mathcal{C}_{s}$ moves vertically with $\mathbf{U}=h_{t} \hat{\mathbf{z}}$, see (12), and $\hat{\mathbf{n}}=\hat{\mathbf{n}}_{s}, d A=d A_{s}$,

$$
U_{n}=u_{n},
$$

on the interior of $\mathcal{A}_{s}$. In this case, (32) is written as

$$
\begin{aligned}
\frac{d E_{m}}{d t} & =\frac{d}{d t} \int_{0}^{h(x, y, t)} d z \int_{B} e_{m} d x d y \\
& =-\int_{\mathcal{A}_{s}} \Delta p_{L} u_{n} d A_{s}+\int_{\partial \mathcal{V}_{r}}\left[\mathbf{T} \cdot \mathbf{u}-\left(e_{m}+p-p_{0}\right) \mathbf{u}\right] \cdot \hat{\mathbf{n}} d A-\dot{\Phi},
\end{aligned}
$$

where $\partial \mathcal{V}_{r}$ denotes the remaining boundary, i.e., the boundary excluding the free surface on which we have used the boundary condition (8), and where we have subtracted the constant $p_{0}$ from the pressure everywhere since $\oint_{\partial \mathcal{V}} \mathbf{u} \cdot \hat{\mathbf{n}} d A=0$ by continuity and incompressibility. To determine the total energy budget, we note that the Laplace pressure acts on both sides of the interface separating 
$\mathcal{A}_{s}$ and $\mathcal{V}$. But the powers it exerts on $\mathcal{A}_{s}$ [see (28)] and on $\mathcal{V}$ [see (34)] differ in their signs and therefore cancel. Since the purely vertical movement of $\mathcal{C}_{s}$ yields

$$
\oint_{\mathcal{C}_{s}} \mathbf{f}_{\gamma} \cdot \mathbf{U} d \mathcal{C}_{s}=\gamma \oint_{\mathcal{C}_{s}} h_{t} \hat{\mathbf{m}}_{s} \cdot \hat{\mathbf{z}} d \mathcal{C}_{s}
$$

[which can also be found from comparing (18) with (16) using (17) for $\mathbf{U}_{C} \equiv \mathbf{0}$ ], we obtain the total energy budget in the form

$$
\frac{d\left(E_{m}+E_{s}\right)}{d t}=\int_{\partial \mathcal{V}_{r}}\left(\mathbf{T} \cdot \mathbf{u}-\left(e_{m}+p-p_{0}\right) \mathbf{u}\right) \cdot \hat{\mathbf{n}} d A-\dot{\Phi}+\gamma \oint_{\mathcal{C}_{s}} h_{t} \hat{\mathbf{m}}_{s} \cdot \hat{\mathbf{z}} d \mathcal{C}_{s} .
$$

For stationary flow, the time derivatives vanish in (36) as does $u_{n}$; see (7). In that case, the total energy budget (36), combining that of the bulk and the surface flow, becomes indistinguishable from that just governing the bulk flow (34). Correspondingly, the only trace of capillarity then comes from the pressure $p$, which must include the Laplace pressure to respect the boundary condition formed by (8) and (9). This is the standard approach, and its validity was recently emphasized in [13] and [14]. If this seems strange, one should keep in mind that surface area is not advected like mass or kinetic energy. In the bulk of the flow, each fluid particle carries a particular mass and kinetic energy with it, but it does not carry a particular surface area with it.

The additional energy term (1) for a static control volume in a steady flow was originally postulated by [11] without explanation. In their follow-up paper [12] they argue that it actually stems from the viscous advection terms in (32). Writing the global stress balance (10) in the form [their Eq. (3.4) in our notation]

$$
\int_{\mathcal{A}_{s}}\left(p-p_{0}\right) \hat{\mathbf{n}}_{s} d A_{s}-\mu \int_{\mathcal{A}_{s}} \hat{\mathbf{n}}_{s} \cdot \mathbf{S} d A_{s}+\gamma \oint_{\mathcal{C}_{s}} \mathbf{d l} \times \hat{\mathbf{n}}_{s}=0,
$$

they then take the inner product with $\mathbf{u}$ inside both integrals to get

$$
\int_{\mathcal{A}_{s}}\left(p-p_{0}\right) \mathbf{u} \cdot \hat{\mathbf{n}}_{s} d A_{s}-\mu \int_{\mathcal{A}_{s}} \mathbf{u} \cdot \mathbf{S} \cdot \hat{\mathbf{n}}_{s} d A_{s}+\gamma \oint_{\mathcal{C}_{s}} \mathbf{u} \cdot\left(\mathbf{d l} \times \hat{\mathbf{n}}_{s}\right)=0 .
$$

Since $\mathbf{u} \cdot \hat{\mathbf{n}}_{s}=0$ (for a stationary flow), they furthermore conclude that

$$
\mu \int_{\mathcal{A}_{s}} \mathbf{u} \cdot \mathbf{S} \cdot \hat{\mathbf{n}}_{s} d A_{s}=\gamma \oint_{\mathcal{C}_{s}} \mathbf{u} \cdot\left(\mathbf{d l} \times \hat{\mathbf{n}}_{s}\right),
$$

relating viscous and capillary effects and reinstating the viscous term that was dropped from the free surface part of the energy equation (36). However, multiplying by $\mathbf{u}$ inside the integral, which might seem a legal operation since the area of integration is arbitrary, is actually illegal since one of the integrals is a loop integral. Rewriting the loop integral in (37) as a surface integral, using the identity (11), we get [as in (10) with (11)]

$$
\int_{\mathcal{A}_{s}}\left[\left(p-p_{0}\right) \hat{\mathbf{n}}_{s}-\mu \mathbf{S} \cdot \hat{\mathbf{n}}_{s}-\gamma\left(\nabla \cdot \hat{\mathbf{n}}_{s}\right) \hat{\mathbf{n}}_{s}\right] d A_{s}=0,
$$

and we can now take $\mathcal{A}_{s}$ as any shape we want and conclude-since the first and last terms are explicitly orthogonal to $\mathbf{u}-$ that

$$
\int_{\mathcal{A}_{s}} \mathbf{u} \cdot \mathbf{S} \cdot \hat{\mathbf{n}}_{s} d A_{s}=0
$$

Indeed, the integrand $\mathbf{u}_{s} \cdot \mathbf{S} \cdot \hat{\mathbf{n}}_{s}$ [which we removed from the free surface to get (36)] must be zero everywhere on the interface, which is simply the shear part of the usual dynamic boundary condition (8) on a free surface, and does not involve the surface tension.

The fact that creation of surface energy through the breakup of intermolecular bonds is a reversible, nondissipative process, was emphasized by Batchelor in 1970, who warned that "changes 
in the surface energy should not be counted as "dissipation"' ([23], p. 570). From a historical perspective, it is interesting that the error of attributing power to the surface tension forces acting on a static control volume was made already by Hagen in the context of Savart sheets, and referenced faithfully by Bouasse in his textbook Jets, Tubes and Canals from 1923 ([24], Sec. 107; see also [25], p. 23). Bouasse noted - perhaps with some scepticism (?)— that the theory predicted a minimum for the thickness profile of the sheet not near the edge, but halfway between the center and the edge.

As we mentioned above, it is perhaps strange that the surface tension forces $\mathbf{f}_{\gamma}$ in (28) do no work when the control volume does not move, since these forces are still acting on a moving fluid. The reason for this is that the surface energy, being proportional to the surface area (13), does not depend on the fluid velocity $\mathbf{u}$. In other words, the surface energy has no kinetic energy term, which is due to the fact that the surface, in our idealized continuum approach, is infinitely thin and thus has no mass. But how can it then have an energy? The reason for this is found in the fact that the surface energy, coming from the breaking of bonds between the liquid molecules, is at least of the order of $k T$ per particle or $R T$ per mol, i.e., around $2 \mathrm{~kJ} / \mathrm{mol}(k, R$, and $T$ denote the Boltzmann, the universal gas constant, and the absolute temperature, respectively), whereas the kinetic energy of matter, say, water, moving at $1 \mathrm{~m} / \mathrm{s}$ is about $0.009 \mathrm{~J} / \mathrm{mol}$. Hence, the ratio of these two energies of around $2 \times 10^{5}$ allows us to neglect the kinetic energy of the organized motion represented by the velocity field $\mathbf{u}$ compared to the surface energy for the same amount of matter-as opposed to the kinetic energy of the random thermal motion, which is also of the order of $k T$ per particle.

Significant parts of this work were funded by the "Austrian COMET Programme" (project: InTribology, Project No. 872176, project coordinator: AC2T research GmbH, Austria) via the Austrian Research Promotion Agency (FFG). T.B. is grateful to Anders Andersen and Alexis Duchesne for many valuable discussions and to Jens Eggers and Emmanuel Villermaux for useful comments. The authors contributed equally to this work.

[1] J. W. S. Rayleigh, On the instability of jets, Proc. London Math. Soc. s1-10, 4 (1878).

[2] L. Rayleigh FRS, On the stability, or instability, of certain fluid motions, Proc. London Math. Soc. s1-11, 57 (1879).

[3] L. D. Landau and B. V. Levich, Dragging of a liquid by a moving plate, Acta Physicochimica USSR 17(1-2), 42 (1942); in Collected Papers of L. D. Landau (Pergamon Press, 1965), p. 355.

[4] G. I. Taylor, The dynamics of thin sheets of fluid. III. Disintegration of fluid sheets, Proc. R. Soc. Lond. A 253, 313 (1959).

[5] F. E. C. Culick, Comments on a ruptured soap film, J. Appl. Phys. 31, 1128 (1960).

[6] F. P. Bretherton, The motion of long bubbles in tubes, J. Fluid. Mech. 10, 166 (1961).

[7] J. Eggers, Nonlinear dynamics and breakup of free-surface flows, Rev. Mod. Phys. 69, 865 (1997).

[8] D. Bonn, J. Eggers, J. Indekeu, J. Meunier, and E. Rolley, Wetting and spreading, Rev. Mod. Phys. 81, 739 (2009).

[9] P.-G. de Gennes, F. Brochard-Wyart, and D. Quéré, Capillarity and Wetting Phenomena (Drops, Bubbles, Pearls, Waves) (Springer, New York, 2004).

[10] G. K. Batchelor, An Introduction to Fluid Dynamics, Cambridge Mathematical Library (2000).

[11] R. K. Bhagat, N. K. Jha, P. F. Linden, and D. I. Wilson, On the origin of the circular hydraulic jump in a thin liquid film, J. Fluid Mech. 851, R5 (2018).

[12] R. K. Bhagat and P. F. Linden, The circular capillary jump, J. Fluid Mech. 896, A25 (2020).

[13] A. Duchesne, A. Andersen, and T. Bohr, Surface tension and the origin of the circular hydraulic jump in a thin liquid film, Phys. Rev. Fluids 4, 084001 (2019).

[14] B. Scheichl, Centred splash of a vertical jet on a horizontal rotating disc: Recent findings and resolving controversies over the hydraulic jump, in Boundary and Interior Layers: Computational and Asymptotic 
Methods (BAIL 2018), Lecture Notes in Computational Science and Engineering, Vol. 135 (Springer, Cham, 2020), p. 201.

[15] L. D. Landau and E. M. Lifshitz, Fluid Dynamics, 2nd ed., Course of Theoretical Physics, Vol. 6 (Pergamon Press, Oxford, New York, 1987).

[16] C. Pozrikidis, Introduction to Theoretical and Computational Fluid Dynamics, 2nd ed. (Oxford University Press, 2011).

[17] B. Chow, P. Lu, and L. Ni, Hamilton's Ricci Flow, Graduate Studies in Mathematics, Vol. 77 (AMS, 2006).

[18] P. Cermelli, E. Fried, and M. E. Gurtin, Transport relations for surface integrals arising in the formulation of balance laws for evolving fluid interfaces, J. Fluid Mech. 544, 339 (2005).

[19] P. Lidström, Moving regions in Euclidean space and Reynolds' transport theorem, Math. Mech. Solids 16, 366 (2011).

[20] J. M. Delhaye, Jump conditions and entropy sources in two-phase systems. Local instant formulation, Int. J. Multiphase Flow 1, 395 (1974).

[21] H. A. Stone, A simple derivation of the time-dependent convective-diffusion equation for surfactant transport along a deforming interface, Phys. Fluids A 2, 111 (1990).

[22] L. G. Leal, Advanced Transport Phenomena: Fluid Mechanics and Convective Transport Processes, Cambridge Series in Mechanical Engineering, Vol. 7 (Cambridge, 2007).

[23] G. K. Batchelor, The stress system in an suspension of force-free particles, J. Fluid Mech. 41, 545 (1970).

[24] H. Bouasse, Jets, Tubes et Canaux (Delagrave, Paris, 1923).

[25] E. Villermaux, Fragmentation versus cohesion, J. Fluid Mech. 898, P1 (2020). 